Supplement of Ocean Sci., 15, 61-73, 2019 https://doi.org/10.5194/os-15-61-2019-supplement (C) Author(s) 2019. This work is distributed under the Creative Commons Attribution 4.0 License.

(c) (1)

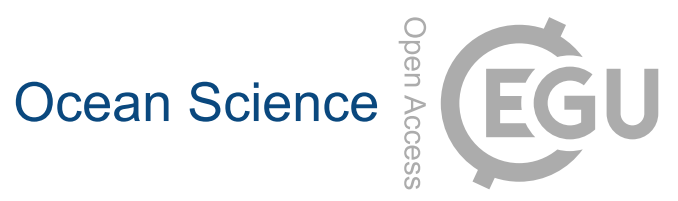

Supplement of

\title{
Measuring rates of present-day relative sea-level rise in low-elevation coastal zones: a critical evaluation
}

Molly E. Keogh and Torbjörn E. Törnqvist

Correspondence to: Molly E. Keogh (mkeogh@tulane.edu)

The copyright of individual parts of the supplement might differ from the CC BY 4.0 License. 
Table S1. Information on 131 tide gauges in coastal Louisiana. Note that several tide gauges have no published benchmarks; in this case, the benchmark setting type and foundation depth are left blank. Other tide gauges have benchmarks with unknown foundations depths. Unknown foundation depth should not be interpreted as foundation depth $=0$.

\begin{tabular}{|c|c|c|c|c|c|c|c|c|c|c|c|c|c|c|c|c|}
\hline $\begin{array}{l}\text { Tide Gauge } \\
\text { Name }\end{array}$ & $\begin{array}{l}\text { Station } \\
\text { Number }\end{array}$ & Agency & Website & $\begin{array}{l}\text { Latitude } \\
\left({ }^{\circ} \mathrm{N}\right)\end{array}$ & $\begin{array}{l}\text { Longitude } \\
\left({ }^{\circ} \mathrm{E}\right)\end{array}$ & $\begin{array}{l}\begin{array}{l}\text { Record } \\
\text { start date }\end{array} \\
\end{array}$ & $\begin{array}{l}\text { Record } \\
\text { end date }\end{array}$ & $\begin{array}{l}\text { Years } \\
\text { active }\end{array}$ & $\begin{array}{l}\begin{array}{l}\text { Number of } \\
\text { published } \\
\text { benchmarks }\end{array} \\
\end{array}$ & $\begin{array}{l}\text { Benchmark } \\
\text { setting type(s) }\end{array}$ & $\begin{array}{l}\text { Benchmark } \\
\text { foundation depths } \\
(\mathrm{m})\end{array}$ & $\begin{array}{l}\text { Depth of } \\
\text { deepest } \\
\text { known } \\
\text { benchmark } \\
\text { foundation } \\
(\mathrm{m})\end{array}$ & $\begin{array}{l}\text { Depth of } \\
\text { shallowest } \\
\text { known } \\
\text { benchmark } \\
\text { foundation } \\
(\mathrm{m}) \\
\end{array}$ & $\begin{array}{l}\text { Depth of } \\
\text { primary } \\
\text { benchmark } \\
\text { foundation } \\
(\mathrm{m})\end{array}$ & $\begin{array}{l}\text { Depth to } \\
\text { Pleistocene } \\
\text { surface }(m)\end{array}$ & $\begin{array}{l}\text { Minimum } \\
\text { benchmark } \\
\text { foundation } \\
\text { height above } \\
\text { Pleistocene } \\
\text { surface }(\mathrm{m}) \\
\end{array}$ \\
\hline Amerada Pass & 8764227 & NOAA & $\begin{array}{l}\text { https:///tidesandcurrents.n } \\
\text { oaa.gov/stationhome.html } \\
\text { ?id=8764227 } \\
\text { https://tidesandcurrents.n } \\
\text { oaa. gov/stationhome.html }\end{array}$ & 29.4500 & -91.3383 & $11 / 1 / 2005$ & $10 / 22 / 2018$ & 12.97 & 9 & $\begin{array}{l}\text { steel rod, concrete } \\
\text { foundation }\end{array}$ & $\begin{array}{l}23,23,23,23,27.4, \\
27.4,27.4 \\
19.5,19.5,19.5\end{array}$ & 27.4 & 23 & 23 & 21 & $\begin{array}{l}\text { Set in } \\
\text { Pleistocene }\end{array}$ \\
\hline Bay Gardene & 8761108 & NOAA & $\begin{array}{l}\text { ?id=8766108 } \\
\text { https://tidesandcurrents.n }\end{array}$ & 29.5983 & -89.6183 & $10 / 16 / 1980$ & $7 / 12 / 1988$ & 7.74 & 5 & steel rod & $19.5,23.2$ & 23.2 & 19.5 & 19.5 & 43 & 20 \\
\hline $\begin{array}{l}\text { Bay Rambo, } \\
\text { Texaco Dock }\end{array}$ & 8761962 & NOAA & $\begin{array}{l}\text { oaa.gov/stationhome.html } \\
\text { ?id =8761962 }\end{array}$ & 29.3617 & -90.1400 & $10 / 29 / 1986$ & $2 / 8 / 1988$ & 1.28 & 5 & steel rod & $\begin{array}{l}24.4,24.4,24.4, \\
24.4,24.4\end{array}$ & 24.4 & 24.4 & 24.4 & 54 & 30 \\
\hline $\begin{array}{l}\text { Bayou St. Denis, } \\
\text { M.V. Petroleum } \\
\text { Dock }\end{array}$ & 8761799 & NOAA & $\begin{array}{l}\text { https://tidesandcurrents.n } \\
\text { oaa.gov/stationhome.html } \\
\text { ?id= }=8761799 \\
\text { https://tidesandcurrents.n } \\
\text { oaa.gov/stationhome.html }\end{array}$ & 29.4967 & -90.0250 & 7/17/1985 & 9/4/1986 & 1.13 & 5 & steel rod & $\begin{array}{l}19.5,21.9,20.7, \\
23.2,21.9\end{array}$ & 23.2 & 19.5 & 19.5 & 44 & 21 \\
\hline Billet Bay & 8761414 & NOAA & $\begin{array}{l}\text { ?id= }=8761414 \\
\text { https://tidesandcurrents.n } \\
\text { oaa.gov/stationhome.html }\end{array}$ & 29.3717 & -89.7517 & $7 / 20 / 1985$ & $11 / 4 / 1986$ & 1.29 & 5 & $\begin{array}{l}\text { steel rod } \\
\text { steel rod, concrete }\end{array}$ & $21.9,20.7$ & 21.9 & 20.7 & 20.7 & 52 & 30 \\
\hline Breton Island & 8760595 & NOAA & ?id $=8760595$ & 29.4933 & -89.1733 & $3 / 17 / 1980$ & $11 / 1 / 1980$ & 0.63 & 6 & monument & $15.2,16.8,18.3$ & 18.3 & 15.2 & 15.2 & 70 & 52 \\
\hline Calcasieu Pass & 8768094 & NOAA & $\begin{array}{l}\text { https://tidesandcurrents.n } \\
\text { oaa.gov/stationhome.html } \\
\text { ?id=8768094 } \\
\text { https://tidesandcurrents.n } \\
\text { oaa.gov/stationhome.html }\end{array}$ & 29.7683 & -93.3433 & $8 / 11 / 2002$ & $10 / 22 / 2018$ & 16.20 & 5 & $\begin{array}{l}\text { fishing pier, large } \\
\text { boulder, davit plate }\end{array}$ & $21.9,15.8,18.3$ & 25 & 24 & $\begin{array}{l}25 \\
\text { unknown } \\
\text { (concrete } \\
\text { retaining }\end{array}$ & 18 & $\begin{array}{l}\text { Set in } \\
\text { Pleistocene }\end{array}$ \\
\hline Caminada Pass & 8761826 & NOAA & $\begin{array}{l}\text { ?id }=861826 \\
\text { https://tidesandcurrents.n }\end{array}$ & 29.2100 & -90.0400 & $10 / 27 / 1986$ & 2/2/1988 & 1.27 & 6 & wall & $18.3,17.1$ & 21.9 & 15.8 & wall) & 55 & Cot in \\
\hline $\begin{array}{l}\text { Chef Menteur } \\
\text { Pass }\end{array}$ & 8761487 & NOAA & $\begin{array}{l}\text { oaa.gov/stationhome.html } \\
\text { ?id = }=86146147 \\
\text { https://tidesandcurrents.n } \\
\text { oaa.gov/stationhome.html }\end{array}$ & 30.0650 & -89.8000 & $11 / 25 / 1982$ & $1 / 2 / 1991$ & 8.10 & 6 & $\begin{array}{l}\text { steel rod, concrete } \\
\text { bridge }\end{array}$ & $\begin{array}{l}23.1,9.1,33.5 \\
35.1,21.9\end{array}$ & 35.1 & 9.1 & 23 & 13 & $\begin{array}{l}\text { Set in } \\
\text { Pleistocene }\end{array}$ \\
\hline Cocodrie & 8762928 & NOAA & $\begin{array}{l}\text { ?id }=862928 \\
\text { https://tidesandcurrents.n } \\
\text { oaa.gov/stationhome.html }\end{array}$ & 29.2450 & -90.6617 & 1/1/1997 & $1 / 1 / 2000$ & 3.00 & 0 & & $16.8,16.8,16.8$ & & & & & \\
\hline Comfort Island & 8760742 & NOAA & $\begin{array}{l}\text { Pid }=86074072 \\
\text { https://tidesandcurrents.n } \\
\text { oaa.gov/stationhome.html }\end{array}$ & 29.8233 & -89.2700 & $3 / 16 / 1980$ & $3 / 3 / 1982$ & 1.96 & 5 & $\begin{array}{l}\text { steel rod } \\
\text { steel rod, } \\
\text { unspecified pipe/rod, }\end{array}$ & $16.8,16.8$ & 16.8 & 16.8 & $\begin{array}{l}16.8 \\
\text { unknown } \\
\text { (unspecified }\end{array}$ & 38 & $\begin{array}{l}21 \\
\text { Set in }\end{array}$ \\
\hline Cypremort Point & 8765251 & NOAA & $\begin{array}{l}\text { ?id }=8765251 \\
\text { https://tidesandcurrents.n } \\
\text { oaa.gov/stationhome.html }\end{array}$ & 29.7133 & -91.8800 & $8 / 8 / 2005$ & $11 / 11 / 2010$ & 5.26 & 5 & seawall & $15.7,19.4$ & 19.4 & 15.7 & rod length) & 10 & Pleistocene \\
\hline Deer Island & 8764101 & NOAA & $? \mathrm{id}=8764101$ & 29.4733 & -91.2667 & $3 / 18 / 1978$ & $11 / 24 / 1978$ & 0.69 & 0 & & & & & unknown & & \\
\hline $\begin{array}{l}\text { Devon Energy } \\
\text { Facility, Pass A } \\
\text { Loutre }\end{array}$ & 8760417 & NOAA & $\begin{array}{l}\text { https://tidesandcurrents.n } \\
\text { oaa. gov/stationhome.html } \\
\text { ?id }=8760417\end{array}$ & 29.2000 & -89.0400 & $4 / 4 / 2008$ & $2 / 10 / 2009$ & 0.85 & 5 & $\begin{array}{l}\text { concrete support } \\
\text { beam } \\
\text { steel rod, concrete }\end{array}$ & unknown & unknown & unknown & $\begin{array}{l}\text { (concrete } \\
\text { support } \\
\text { beam) }\end{array}$ & & \\
\hline $\begin{array}{l}\text { East Bay, Shell } \\
\text { Oil } \\
\text { East Isle } \\
\text { Dernieres, Lake }\end{array}$ & 8760781 & NOAA & $\begin{array}{l}\text { https://tidesandcurrents.n } \\
\text { oaa.gov/stationhome.html } \\
\text { ?id=87600781 } \\
\text { https:///tidesandcurrents.n } \\
\text { oaa.gov/stationhome.html }\end{array}$ & 29.0533 & -89.3050 & $1 / 8 / 1985$ & $2 / 20 / 1986$ & 1.12 & 6 & $\begin{array}{l}\text { platform, concrete } \\
\text { pier, concrete } \\
\text { foundation }\end{array}$ & 14.6 & 14.6 & 14.6 & $\begin{array}{l}\text { unknown } \\
\text { (concrete } \\
\text { platform) }\end{array}$ & 106 & 91 \\
\hline Pelto & 8762888 & NOAA & $\begin{array}{l}\text { ?id }=862888 \\
\text { https: //tidesandcurrents.n }\end{array}$ & 29.0717 & -90.6400 & $10 / 30 / 1986$ & 9/9/1993 & 6.86 & 0 & & & & & & & \\
\hline $\begin{array}{l}\text { East Timbalier } \\
\text { Island }\end{array}$ & 8762223 & NOAA & $\begin{array}{l}\text { oaa.gov/stationhome.html } \\
\text { ?id = } 8762223 \text { t\#ides } \\
\text { https://tidesandcurrents.n }\end{array}$ & 29.0767 & -90.2850 & $10 / 28 / 1986$ & $1 / 6 / 1988$ & 1.19 & 5 & steel rod & $\begin{array}{l}20.7,21.9,23.9, \\
28.8,21.9\end{array}$ & 28.8 & 20.7 & 20.7 & 46 & 17 \\
\hline $\begin{array}{l}\text { Canal } \\
\text { Cantilut }\end{array}$ & 8761207 & NOAA & 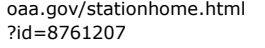 & 29.3750 & -89.6017 & $7 / 23 / 1985$ & $1 / 2 / 1991$ & 5.45 & 0 & & & & & & & \\
\hline $\begin{array}{l}\text { Equitable } \\
\text { Petroleum Dock, } \\
\text { Turtle Bay }\end{array}$ & 8761959 & NOAA & $\begin{array}{l}\text { https://tidesandcurrents.n } \\
\text { oaa.gov/stationhome.html } \\
\text { ?idd=8761959 } \\
\text { https://tidesandcurrents.n } \\
\text { oaa.gov/stationhome.html }\end{array}$ & 29.5200 & -90.1383 & $10 / 29 / 1986$ & 6/13/1987 & 0.62 & 0 & & & & & & & \\
\hline Eugene Island & 8764311 & NOAA & $\begin{array}{l}\text { ?idt=8764311 } \\
\text { https://tidesandcurrents.n } \\
\text { oaa.gov/stationhome.html }\end{array}$ & 29.3717 & -91.3850 & 6/1/1939 & $8 / 16 / 2005$ & 66.21 & 0 & & & & & & & \\
\hline North of & 8764314 & NOAA & $? i d=8764314$ & 29.3667 & -91.3833 & $11 / 15 / 2014$ & $10 / 22 / 2018$ & 3.93 & 0 & & & & & & & \\
\hline
\end{tabular}




\begin{tabular}{|c|c|c|c|c|c|c|c|c|c|c|c|c|c|c|c|c|}
\hline $\begin{array}{l}\text { Fourchon } \\
\text { (LUMCON) }\end{array}$ & 8762079 & NOAA & $\begin{array}{l}\text { https://tidesandcurrents.n } \\
\text { oaa.gov/stationhome.html } \\
\text { ?id=8762079 }\end{array}$ & 29.1133 & -90.1850 & 10/10/1986 & $6 / 6 / 1988$ & 1.66 & 0 & & & & & & & \\
\hline Freshwater & & & $\begin{array}{l}\text { https://tidesandcurrents.n } \\
\text { oaa.gov/stationhome.html } \\
\text { Jid=8766072 }\end{array}$ & & & & & & & $\begin{array}{l}\text { steel rod, concrete } \\
\text { structure, brass bolt } \\
\text { set in support wall }\end{array}$ & & & & $\begin{array}{l}\text { unknown } \\
\text { (cement } \\
\text { structure) }\end{array}$ & & $\begin{array}{l}\text { Set in } \\
\text { Pleistocene }\end{array}$ \\
\hline Canal Locks & 8766072 & NOAA & $\begin{array}{l}\text { ?id }=8766072 \\
\text { https://tidesandcurrents.n } \\
\text { oaa.gov/stationhome.html }\end{array}$ & 29.5517 & -92.3050 & $8 / 3 / 2005$ & $10 / 22 / 2018$ & 13.22 & 4 & & $15.3,17.1$ & 17.1 & 15.3 & & 15 & Pleistocene \\
\hline Grand Isle & 8761720 & NOAA & $\begin{array}{l}\text { ?id }=8761720 \\
\text { https://tidesandcurrents.n } \\
\text { oaa.gov/stationhome.html }\end{array}$ & 29.2550 & -89.9683 & 8/2/1947 & $5 / 14 / 1980$ & 32.78 & 0 & $\begin{array}{l}\text { steel rod, concrete } \\
\text { seawall, concrete }\end{array}$ & & & & $\begin{array}{l}\text { unknown } \\
\text { (concrete }\end{array}$ & & \\
\hline Grand Isle & 8761724 & NOAA & $\begin{array}{l}\text { ?id }=8761724 \\
\text { https://tidesandcurrents.n }\end{array}$ & 29.2633 & -89.9567 & 11/9/1979 & $10 / 22 / 2018$ & 38.95 & 5 & bulkhead & $18.3,19.8$ & 19.8 & 18.3 & seawall) & 57 & 37 \\
\hline Grand Pass & 8760668 & NOAA & $\begin{array}{l}\text { oaa.gov/stationhome.html } \\
\text { ?idt=8760668 } \\
\text { https://tidesandcurrents.n }\end{array}$ & 30.1267 & -89.2217 & 12/10/1980 & $4 / 3 / 1996$ & 15.31 & 5 & steel rod & $\begin{array}{l}18.3,16.8,19.8 \\
18.3,23.2\end{array}$ & 23.2 & 16.8 & 18.3 & 15 & $\begin{array}{l}\text { Set in } \\
\text { Pleistocene }\end{array}$ \\
\hline Greens Ditch & 8761426 & NOAA & $\begin{array}{l}\text { oaa.gov/stationhome.html } \\
\text { ?id }=8761426 \\
\text { https://tidesandcurrents.n }\end{array}$ & 30.1117 & -89.7600 & 11/25/1982 & 10/20/1983 & 0.90 & 6 & $\begin{array}{l}\text { steel rod, concrete } \\
\text { monument }\end{array}$ & $\begin{array}{l}18.3,19.5,19.5 \\
21.9,20.7\end{array}$ & 21.9 & 18.3 & 18.3 & 8 & $\begin{array}{l}\text { Set in } \\
\text { Pleistocene }\end{array}$ \\
\hline $\begin{array}{l}\text { Hackberry Bay, } \\
\text { Texaco Dock }\end{array}$ & 8761819 & NOAA & $\begin{array}{l}\text { oaa.gov/stationhome.html } \\
\text { ?id=8761819 } \\
\text { https://tidesandcurrents.n }\end{array}$ & 29.4017 & -90.0383 & 10/28/1986 & $11 / 13 / 1987$ & 1.04 & 5 & steel rod & $\begin{array}{l}25.6,29.3,30.5, \\
24.4,28.0\end{array}$ & 30.5 & 24.4 & 25.6 & 52 & 22 \\
\hline $\begin{array}{l}\text { I-10 Bonnet } \\
\text { Carre Floodway } \\
\text { Lafitte, } \\
\text { Barataria }\end{array}$ & 8762483 & NOAA & $\begin{array}{l}\text { oaa.gov/stationhome.html } \\
\text { ?idd=8762483 } \\
\text { https://tidesandcurrents.n } \\
\text { oaa.gov/stationhome.html }\end{array}$ & 30.0683 & -90.3900 & 4/8/2015 & $10 / 22 / 2018$ & 3.54 & 0 & $\begin{array}{l}\text { steel rod, concrete } \\
\text { foundation, concrete }\end{array}$ & & & & $\begin{array}{l}\text { unknown } \\
\text { (concrete }\end{array}$ & & \\
\hline $\begin{array}{l}\text { Barataria } \\
\text { Waterway } \\
\text { Lake Judge }\end{array}$ & 8761899 & NOAA & $\begin{array}{l}\text { oaa.gov/stationhome.html } \\
\text { ?id=8761899 }\end{array}$ & 29.6667 & -90.1117 & 6/29/1985 & $1 / 2 / 1991$ & 5.51 & 7 & & $\begin{array}{l}24.4,18.3,19.5, \\
30.5\end{array}$ & 30.5 & 18.3 & foundation) & 37 & 7 \\
\hline $\begin{array}{l}\text { Perez, } \\
\text { Hermitage }\end{array}$ & & & $\begin{array}{l}\text { https://tidesandcurrents.n } \\
\text { oaa.gov/stationhome.html }\end{array}$ & & & & & & & & $20.7,24.4,4.9$ & & & & & \\
\hline Leeville, Bayou & 8101002 & IVUAA & $\begin{array}{l}\text { https://tidesandcurrents.n } \\
\text { oaa.gov/stationhome.html }\end{array}$ & 29.5583 & -89.8833 & $6 / 29 / 1985$ & 10/1/1986 & 1.26 & 7 & steel rod, concrete & $\begin{array}{l}23.2,21.9 \\
18,20,24,20,20,\end{array}$ & 24.4 & 4.9 & 20.7 & 39 & 15 \\
\hline Lafourche & 8762084 & NOAA & $\begin{array}{l}\text { ?id }=8762084 \\
\text { https://tidesandcurrents.n } \\
\text { oaa. gov/stationhome.html }\end{array}$ & 29.2483 & -90.2117 & 11/1/1986 & $1 / 8 / 1991$ & 4.19 & 10 & monument & 28,5 & 28 & 5 & 18 & 57 & 29 \\
\hline Atchafalaya Bay & 8765026 & NOAA & $? \mathrm{id}=8765026$ & 29.4850 & -91.7633 & $6 / 1 / 1960$ & $10 / 27 / 1978$ & 18.40 & 0 & & & & & unknown & & \\
\hline Martello Castle & 8761529 & NOAA & $\begin{array}{l}\text { https://tidesandcurrents.n } \\
\text { oaa.gov/stationhome.html } \\
\text { ?idt=8761529 } \\
\text { https://tidesandcurrents.n }\end{array}$ & 29.9450 & -89.8350 & $12 / 6 / 2006$ & $6 / 6 / 2007$ & 0.50 & 4 & $\begin{array}{l}\text { steel rod, concrete } \\
\text { monument }\end{array}$ & $18.90,19.51$ & 19.51 & 18.90 & $\begin{array}{l}\text { (rough } \\
\text { poured } \\
\text { concrete) }\end{array}$ & 19 & $\begin{array}{l}\text { Set in } \\
\text { Pleistocene }\end{array}$ \\
\hline $\begin{array}{l}\text { Mendicant } \\
\text { Island }\end{array}$ & 8761742 & NOAA & $\begin{array}{l}\text { oaa. gov/stationhome.html } \\
\text { ?id=8761742 } \\
\text { https://tidesandcurrents.n }\end{array}$ & 29.3183 & -89.9800 & $7 / 19 / 1985$ & 9/4/1986 & 1.13 & 5 & steel rod & $\begin{array}{l}24.4,24.4,21.9 \\
23.2,20.7\end{array}$ & 24.4 & 20.7 & 24.4 & 55 & 31 \\
\hline $\begin{array}{l}\text { North Pass } \\
\text { Olga } \\
\text { Compressor }\end{array}$ & 8760412 & NOAA & $\begin{array}{l}\text { oaa.gov/stationhome.html } \\
\text { ?id }=8760412 \\
\text { https://tidesandcurrents.n } \\
\text { oaa.gov/stationhome.html }\end{array}$ & 29.2050 & -89.0367 & $3 / 20 / 1980$ & $11 / 1 / 1980$ & 0.62 & 5 & steel rod & $\begin{array}{l}\text { l5.2, 15.2, 15.2, } \\
15.2,15.2\end{array}$ & 15.2 & 15.2 & $\begin{array}{l}15.2 \\
\text { unknown } \\
\text { (compressor }\end{array}$ & 142 & 127 \\
\hline Station & 8760889 & NOAA & $\begin{array}{l}\text { ?id }=8760889 \\
\text { https://tidesandcurrents.n } \\
\text { oga, gov/stationhome html }\end{array}$ & 29.3867 & -89.3800 & $7 / 12 / 1985$ & $2 / 1 / 2009$ & 23.56 & 5 & concrete platform & unknown & unknown & unknown & platform) & & \\
\hline Pass Manchac & 8762273 & NOAA & $\begin{array}{l}\text { oaa.gov/stationhome.html } \\
\text { ?idt=8762273 } \\
\text { https://tidesandcurrents.n }\end{array}$ & 30.2967 & -90.3117 & $11 / 23 / 1982$ & $6 / 7 / 1988$ & 5.54 & 4 & steel rod & $\begin{array}{l}\begin{array}{l}19.5,20.7,19.5, \\
19.5\end{array} \\
\end{array}$ & 20.7 & 19.5 & 19.5 & 15 & Pleistocene \\
\hline $\begin{array}{l}\text { Pelican Island, } \\
\text { Chevron Station }\end{array}$ & 8761198 & NOAA & $\begin{array}{l}\text { oaa.gov/stationhome.html } \\
\text { ?id= }=8761198 \\
\text { https://tidesandcurrents.n }\end{array}$ & 29.2667 & -89.5983 & 7/9/1985 & 9/3/1986 & 1.15 & 5 & steel rod & $\begin{array}{l}20.7,21.9,19.5 \\
19.5,19.5\end{array}$ & 21.9 & 19.5 & 20.7 & 64 & 42 \\
\hline $\begin{array}{l}\text { lilots Station } \\
\text { East }\end{array}$ & 8760922 & NOAA & $\begin{array}{l}\text { oaa.gov/statitonhome.html } \\
\text { ?id=8760922 } \\
\text { https://tidesandcurrents.n } \\
\text { oaa.gov/stationhome.html }\end{array}$ & 28.9317 & -89.4067 & $3 / 8 / 2004$ & $10 / 22 / 2018$ & 14.62 & 0 & $\begin{array}{l}\text { steel rod, concrete } \\
\text { walkway, concrete }\end{array}$ & & & & & & \\
\hline Pilottown & 8760721 & NOAA & $\begin{array}{l}\text { ?id }=8760721 \\
\text { https://tidesandcurrents.n } \\
\text { oaa. gov/stationhome.html }\end{array}$ & 29.1783 & -89.2583 & $12 / 19 / 1980$ & $10 / 22 / 2018$ & 37.84 & 5 & helipad & $32.0,20$ & 32 & 32.0 & 32 & 88 & 56 \\
\hline $\begin{array}{l}\text { Point au Fer } \\
\text { Point Barre, }\end{array}$ & 8764256 & NOAA & $\begin{array}{l}\text { ?id }=8764256 \\
\text { https://tidesandcurrents.n } \\
\text { oaa.gov/stationhome.html }\end{array}$ & 29.3333 & -91.3533 & 6/1/1960 & $11 / 17 / 1978$ & 18.46 & 0 & & & & & & & \\
\hline Humble Canal & 8762731 & NOAA & $\begin{array}{l}\text { ?id }=8762731 \\
\text { https://tidesandcurrents.n } \\
\text { oaa gov/stationhome htm }\end{array}$ & 29.4283 & -90.5567 & 10/25/1986 & $11 / 3 / 1987$ & 1.02 & 0 & & & & & & & \\
\hline $\begin{array}{l}\text { Cut Off Canal } \\
\text { Cont }\end{array}$ & 8762525 & NOAA & $\begin{array}{l}\text { aaa.gov//stationhome.html } \\
\text { ?id =8762525 } \\
\text { https://tidesandcurrents.n }\end{array}$ & 29.4167 & -90.4467 & 4/30/1987 & $8 / 2 / 1988$ & 1.26 & 0 & & & & & & & \\
\hline $\begin{array}{l}\text { Pointe au Chien, } \\
\text { St. Louis Canal }\end{array}$ & 8762571 & NOAA & $\begin{array}{l}\text { oaa.gov/stationhome.html } \\
\text { ?id = } 8762571 \\
\text { https://tidesandcurrents.n }\end{array}$ & 29.4567 & -90.4717 & 10/7/1986 & $3 / 2 / 1988$ & 1.40 & 0 & & & & & & & \\
\hline $\begin{array}{l}\text { Port Fourchon, } \\
\text { Belle Pass }\end{array}$ & 8762075 & NOAA & $\begin{array}{l}\text { oaa.gov//stationhome.html } \\
\text { ?id=8762075 }\end{array}$ & 29.1133 & -90.1983 & $7 / 19 / 2003$ & $10 / 22 / 2018$ & 15.26 & 0 & & & & & & & \\
\hline
\end{tabular}




\begin{tabular}{|c|c|c|c|c|c|c|c|c|c|c|c|c|c|c|c|c|}
\hline $\begin{array}{l}\text { Racoon Point, } \\
\text { Isle Dernieres }\end{array}$ & 8763506 & NOAA & $\begin{array}{l}\text { https://tidesandcurrents.n } \\
\text { oaa.gov/stationhome.html } \\
\text { ?id=8763506 }\end{array}$ & 29.0633 & -90.9617 & $2 / 1 / 1979$ & $5 / 10 / 1979$ & 0.27 & 0 & & & & & & & \\
\hline & & & $\begin{array}{l}\text { https://tidesandcurrents.n } \\
\text { oaa. gov/stationhome.html }\end{array}$ & & & & & & & $\begin{array}{l}\text { concrete pad, } \\
\text { concrete monument, } \\
\text { concrete bridge } \\
\text { abutment, concrete }\end{array}$ & & & & $\begin{array}{l}\text { unknown } \\
\text { (concrete }\end{array}$ & & \\
\hline The Rigolets & 8761402 & NOAA & $\begin{array}{l}\text { ?id }=8761402 \\
\text { https://tidesandcurrents.n } \\
\text { oaa.gov/stationhome.html }\end{array}$ & 30.1667 & -89.7367 & $12 / 4 / 1980$ & $11 / 22 / 2014$ & 33.97 & 5 & stair & unknown & unknown & unknown & & & \\
\hline Salt Point & 8764632 & NOAA & $? \mathrm{id}=8764632$ & 29.5733 & -91.5367 & $5 / 15 / 1978$ & $7 / 11 / 1978$ & 0.16 & 0 & & & & & & & \\
\hline & & & $\begin{array}{l}\text { https://tidesandcurrents.n } \\
\text { oaa.gov/stationhome.html } \\
\text { ?id }=8761305\end{array}$ & & & & & & & $\begin{array}{l}\text { steel rod, concrete } \\
\text { foundation, raised }\end{array}$ & $\begin{array}{l}27.4,27.4,27.4, \\
27.4,27.4,27.4,\end{array}$ & & & & & $\begin{array}{l}\text { Set in } \\
\text { Pleistocene }\end{array}$ \\
\hline Shell Beach & 8761305 & NOAA & $\begin{array}{l}\text { ?id }=8761305 \\
\text { https://tidesandcurrents.n } \\
\text { oaa.gov/stationhome.html }\end{array}$ & 29.8683 & -89.6733 & $11 / 7 / 1979$ & $10 / 22 / 2018$ & 38.96 & 11 & & & 27.4 & 27.4 & 27.4 & 27 & Pleistocene \\
\hline Atchafalaya Bay & 8764165 & NOAA & $\begin{array}{l}\text { ?id }=8764165 \\
\text { https://tidesandcurrents.n } \\
\text { oaa.gov/stationhome.html }\end{array}$ & 29.4733 & -91.3050 & $6 / 1 / 1960$ & $6 / 1 / 1961$ & 1.00 & 0 & & & & & & & \\
\hline South Pass & 8760550 & NOAA & $\begin{array}{l}\text { ?id }=8760550 \\
\text { https://tidesandcurrents.n } \\
\text { oaa.gov/stationhome.html }\end{array}$ & 28.9917 & -89.1400 & $3 / 8 / 1981$ & 10/15/1984 & 3.61 & 0 & & & & & & & \\
\hline South Pass & 8760551 & NOAA & $\begin{array}{l}\text { ?id= }=8760551 \\
\text { https://tidesandcurrents.n } \\
\text { oaa.gov/stationhome.html }\end{array}$ & 28.9900 & -89.1400 & $11 / 8 / 1977$ & $9 / 26 / 2000$ & 22.88 & 0 & & 24.4, 24.4, 24.4, & & & & & \\
\hline Southwest Pass & 8760943 & NOAA & $\begin{array}{l}\text { ?id }=8760943 \\
\text { htttps://tidesandcurrents.n } \\
\text { oaa.gov/stationhome.html }\end{array}$ & 28.9250 & -89.4183 & 5/27/1996 & $3 / 8 / 2004$ & 7.78 & 4 & steel rod & $\begin{array}{l}24.4 \\
19.5,20.7,20.7,\end{array}$ & 24.4 & 24.4 & 24.4 & 109 & 85 \\
\hline $\begin{array}{l}\text { St. Mary's Point } \\
\text { Superior Oil, } \\
\text { Four Island }\end{array}$ & 8761679 & NOAA & $\begin{array}{l}\text { ?id }=8761679 \\
\text { https://tidesandcurrents.n } \\
\text { oaa. gov/stationhome.html }\end{array}$ & 29.4317 & -89.9383 & $7 / 18 / 1985$ & $1 / 6 / 1987$ & 1.47 & 5 & steel rod & $23.2,24.4$ & 24.4 & 19.5 & 19.5 & 50 & 26 \\
\hline $\begin{array}{l}\text { Bayou } \\
\text { Texaco Gas } \\
\text { Platform, Caillou }\end{array}$ & 8763153 & NOAA & $\begin{array}{l}\text { ?id }=8763153 \\
\text { https://tidesandcurrents.n } \\
\text { oaa.gov/stationhome.html }\end{array}$ & 29.2350 & -90.7800 & $10 / 30 / 1986$ & $11 / 19 / 1987$ & 1.05 & 0 & concrete platform, & & & & $\begin{array}{l}\text { unknown } \\
\text { (concrete }\end{array}$ & & \\
\hline $\begin{array}{l}\text { Bay } \\
\text { Texaco TB\#3, } \\
\text { Bayou Petit }\end{array}$ & 8763535 & NOAA & $\begin{array}{l}\text { ?id }=8763535 \\
\text { https://tidesandcurrents.n } \\
\text { oaa. gov/stationhome.html }\end{array}$ & 29.1750 & -90.9767 & $10 / 11 / 2008$ & $12 / 18 / 2012$ & 4.19 & 5 & concrete pier & unknown & unknown & unknown & platform) & & \\
\hline Caillou & 8762938 & NOAA & $\begin{array}{l}\text { ?id=8766938 } \\
\text { https://tidesandcurrents.n } \\
\text { oaa. gov/stationhome.html }\end{array}$ & 29.1867 & -90.6667 & $10 / 22 / 1986$ & $4 / 7 / 1988$ & 1.46 & 0 & & & & & & & \\
\hline Timbalier Island & 8762582 & NOAA & $? \mathrm{id}=8762582$ & 29.0667 & -90.4767 & 10/29/1986 & $3 / 1 / 1988$ & 1.34 & 0 & & & & & & & \\
\hline Vicinity of UNO & & & $\begin{array}{l}\text { https://tidesandcurrents.n } \\
\text { oaa.gov/stationhome.html }\end{array}$ & & & & & & & $\begin{array}{l}\text { concrete bridge } \\
\text { abutment, concrete } \\
\text { seawall cap, }\end{array}$ & & & & $\begin{array}{l}\text { Unknown } \\
\text { (bridge } \\
\text { abutment } \\
\text { wing wall }\end{array}$ & & \\
\hline Chef Menteur & 8761385 & NOAA & $\begin{array}{l}\text { ?id }=8761385 \\
\text { https://tidesandcurrents.n } \\
\text { oaa.gov/stationhome.html }\end{array}$ & 30.0683 & -89.8017 & 9/26/2014 & $11 / 21 / 2014$ & 0.15 & 5 & concrete monument & unknown & unknown & unknown & wing wall) & & \\
\hline Wax Lake Outlet & 8764361 & NOAA & $\begin{array}{l}\text { ?id=87646361 } \\
\text { https://tidesandcurrents.n } \\
\text { oaa. gov/stationhome.html }\end{array}$ & 29.5733 & -91.4200 & 4/4/1978 & $11 / 24 / 1978$ & 0.64 & 0 & & & & & $\begin{array}{l}\text { unknown } \\
\text { (unspecified }\end{array}$ & & Set in \\
\hline $\begin{array}{l}\text { Mermentau } \\
\text { River at Grand } \\
\text { Chenieer (sic) }\end{array}$ & 8765148 & NOAA & $\begin{array}{l}\text { ?id=8765148 } \\
\text { http://rivergages.mvr.usac } \\
\text { e.army.mil/WaterControl/s } \\
\text { tationinfo2.cfm?sid }=70900 \\
\text { \&fid }=8 \mathrm{dt}=\mathrm{S}\end{array}$ & 29.8367 & -91.8367 & $8 / 19 / 2001$ & 9/19/2001 & 0.08 & at least 1 & steel rod & $12.8,14.30$ & 14.3 & 12.8 & $\begin{array}{l}\text { rod length) } \\
\text { unknown } \\
\text { (primary } \\
\text { benchmark } \\
\text { not }\end{array}$ & 5 & Pleistocene \\
\hline $\begin{array}{l}\text { Barataria } \\
\text { Waterway at }\end{array}$ & 70900 & USACE & $\begin{array}{l}\text { \&fid=\&dt=S } \\
\text { http://rivergages.mvr.usac } \\
\text { e.army.mil/WaterControl/s } \\
\text { tationinfo2.cfm?sid }=82875\end{array}$ & 29.7704 & -93.0135 & 2/24/2012 & $10 / 22 / 2018$ & 6.66 & at least 1 & steel rod & 1.5 & 1.5 & 1.5 & $\begin{array}{l}\text { specified) } \\
\text { unknown } \\
\text { (primary } \\
\text { benchmark } \\
\text { not }\end{array}$ & 6 & 5 \\
\hline $\begin{array}{l}\text { Bayou Petit } \\
\text { Caillou at }\end{array}$ & 82875 & USACE & $\begin{array}{l}\text { \&fid=\&dt=S } \\
\text { http://rivergages.mvr.usac } \\
\text { e.army.mil/WaterControl/s } \\
\text { tationinfo2.cfm?sid }=76305\end{array}$ & 29.6694 & -90.1106 & 2/18/2003 & $10 / 22 / 2018$ & 15.67 & at least 2 & steel rod & 7.4 & 7.4 & 7.4 & $\begin{array}{l}\text { specified) } \\
\text { unknown } \\
\text { (primary } \\
\text { benchmark } \\
\text { not }\end{array}$ & 36 & 29 \\
\hline $\begin{array}{l}\text { East Cote } \\
\text { Blanche Bay at }\end{array}$ & 76305 & USACE & $\begin{array}{l}\text { \&ffid=\&dd=S } \\
\text { http://rivergages.mvr.usac } \\
\text { e.army.mil/Watercontrol/s } \\
\text { tationinfo2.cfm?sid=880800 }\end{array}$ & 29.2543 & -90.6635 & 3/5/1969 & $12 / 31 / 2014$ & 45.82 & at least 1 & steel rod & 24.4 & 24.4 & 24.4 & specified) & 57 & 33 \\
\hline Luke's Landing & 88800 & USACE & \&fid $=\& d t=S$ & -91.5400 & -91.5400 & 2/22/1957 & $10 / 2 / 2002$ & 45.61 & 0 & & & & & & & \\
\hline $\begin{array}{l}\text { Crewboat } \\
\text { Channel at Wax } \\
\text { Lake Outlet near } \\
\text { Calumet }\end{array}$ & 03830 & USACE & $\begin{array}{l}\text { http://rivergages.mvr.usac } \\
\text { e.army.mil/WaterControl/s } \\
\text { tationinfo2.cfm?sid=03830 } \\
\text { \&fid=\&dt=S\&pcode=HG }\end{array}$ & 29.5400 & -91.4356 & 4/14/1973 & $10 / 22 / 2018$ & 45.52 & 0 & & & & & & & \\
\hline
\end{tabular}




\begin{tabular}{|c|c|c|c|c|c|c|c|c|c|c|c|c|c|c|}
\hline $\begin{array}{l}\text { Avoca Island } \\
\text { Cutoff south of }\end{array}$ & & & $\begin{array}{l}\text { http:///rivergages.mvr.usac } \\
\text { e.army.mil/WaterControl/s } \\
\text { tationinfo2.cf? } 2 \text { sid }=03820\end{array}$ & & & & & & & & & & & $\begin{array}{l}\text { unknown } \\
\text { (primary } \\
\text { benchmark } \\
\text { not }\end{array}$ \\
\hline Morgan City & 03820 & USACE & \&fid $=\& d t=S \& p c o d e=H G$ & 29.5333 & -91.2494 & 2/29/1956 & $3 / 12 / 2015$ & 59.03 & at least 1 & regular concrete & unknown & unknown & unknown & $\begin{array}{l}\text { specified) } \\
\text { unknown }\end{array}$ \\
\hline $\begin{array}{l}\text { Golden Meadow } \\
\text { Floodgate }\end{array}$ & & & $\begin{array}{l}\text { http:///rivergages.mur.usac } \\
\text { e.army.mil/waterControl/s } \\
\text { tationinfo2.cfm?sid }=82250\end{array}$ & & & & & & & & & & & $\begin{array}{l}\text { (primary } \\
\text { benchmark } \\
\text { not }\end{array}$ \\
\hline (North) & 82250 & USACE & $\begin{array}{l}\text { \&fid=\&dt=S\&pcode=HG } \\
\text { http://rivergages.mur.usac } \\
\text { e.army.mil/WaterControl/s }\end{array}$ & 29.3442 & -90.2464 & $3 / 16 / 2004$ & $5 / 8 / 2014$ & 10.14 & at least 1 & concrete headwall & unknown & unknown & unknown & $\begin{array}{l}\text { Specified) } \\
\text { unknown } \\
\text { (primary } \\
\text { benchmark }\end{array}$ \\
\hline $\begin{array}{l}\begin{array}{l}\text { Floodgate } \\
\text { (South) }\end{array} \\
\text { (S) }\end{array}$ & 82260 & USACE & $\begin{array}{l}\text { tationinfo2.cfm?sid }=82260 \\
\text { \&fid }=\& d t=S \& \text { pcode }=H G\end{array}$ & 29.3425 & -90.2464 & $3 / 16 / 2004$ & $5 / 8 / 2014$ & 10.14 & at least 1 & concrete headwall & unknown & unknown & unknown & $\begin{array}{l}\text { not } \\
\text { specified) } \\
\text { unknown } \\
\text { (primary }\end{array}$ \\
\hline $\begin{array}{l}\text { Bayou Des } \\
\text { Allemands at } \\
\text { Des Allemands }\end{array}$ & 82700 & USACE & $\begin{array}{l}\text { e.army.mil/WaterControl/s } \\
\text { tationinfo2.cfm?sid }=82700 \\
\text { \&fid=\&dt=S\&pcode }=\text { HG }\end{array}$ & 29.8239 & -90.4767 & $5 / 22 / 2009$ & $10 / 22 / 2018$ & 9.42 & at least 1 & concrete monument & unknown & unknown & unknown & $\begin{array}{l}\text { benchmark } \\
\text { not } \\
\text { specified) } \\
\text { unknown }\end{array}$ \\
\hline $\begin{array}{l}\text { Mississippi River } \\
\text { South Pass at }\end{array}$ & & & $\begin{array}{l}\text { http: ///rivergages.mvr.usac } \\
\text { e.army.mil/Watercontrol/s } \\
\text { tationinfo2.cfm?sid=01850 }\end{array}$ & & & & & & & & & & & $\begin{array}{l}\text { (primary } \\
\text { benchmark } \\
\text { not }\end{array}$ \\
\hline Port Eads & 01850 & USACE & \&fid $=\& d t=S$ & 28.9323 & -89.4071 & $1 / 1 / 1950$ & 7/7/2015 & 65.51 & at least 2 & steel rod & 0.9 & 0.9 & 0.9 & specified) \\
\hline $\begin{array}{l}\text { Lake } \\
\text { Pontchartrain at } \\
\text { Frenier }\end{array}$ & 85550 & USACE & $\begin{array}{l}\text { http://rivergages.mvr.usac } \\
\text { e.army.mil//WaterControl/s } \\
\text { tationinfo2.cfm?sid=85550 } \\
\text { \&fid=\&dt=S\&pcode = HG } \\
\text { http://rivergages.mvr.usac }\end{array}$ & 30.1061 & -90.4214 & $1 / 26 / 1950$ & $6 / 4 / 2005$ & 55.35 & 0 & & & & & \\
\hline $\begin{array}{l}\text { Calcasieu River } \\
\text { and Pass near }\end{array}$ & & & $\begin{array}{l}\text { e.army.mil/WaterControl/s } \\
\text { tationinfo2.cfm?sid }=73650\end{array}$ & & & & & & & & & & & \\
\hline $\begin{array}{l}\text { Cameron } \\
\text { rresnwater }\end{array}$ & 73650 & USACE & $\&$ fid $=\& d t=S$ & 29.7758 & -93.3479 & 1/1/1955 & $8 / 14 / 2005$ & 50.62 & unknown & unknown & unknown & unknown & unknown & unknown \\
\hline $\begin{array}{l}\text { Canal at } \\
\text { Freshwater } \\
\text { Bayou Lock - }\end{array}$ & & & $\begin{array}{l}\text { http:///rivergages.mvr.usac } \\
\text { e.army.mil/Watercontrol/s } \\
\text { tationinfo2.cfm?sid=76592 }\end{array}$ & & & & & & & & & & & \\
\hline $\begin{array}{l}\text { North } \\
\text { rreshwater }\end{array}$ & 76592 & USACE & \&fid $=\& d t=S$ & 29.5547 & -92.3046 & $3 / 15 / 2004$ & $10 / 22 / 2018$ & 14.60 & unknown & unknown & unknown & unknown & unknown & unknown \\
\hline $\begin{array}{l}\text { Canal at } \\
\text { Freshwater } \\
\text { Bayou Lock - }\end{array}$ & & & $\begin{array}{l}\text { http://rivergages.mvr.usac } \\
\text { e.army.mi//WaterControl/s } \\
\text { tationinfo2.cfm?sid=76593 }\end{array}$ & & & & & & & & & & & \\
\hline South & 76593 & USACE & $\begin{array}{l}\text { \&fid=\&dt=S } \\
\text { http://rivergages.mvr.usac }\end{array}$ & 29.5524 & -92.3050 & $7 / 28 / 1968$ & $10 / 21 / 2012$ & 44.23 & unknown & unknown & unknown & unknown & unknown & unknown \\
\hline $\begin{array}{l}\text { Schooner Bayou } \\
\text { Control }\end{array}$ & & & $\begin{array}{l}\text { e.army.mil/WaterControl/s } \\
\text { tationinfo2.cfm?sid=76680 }\end{array}$ & & & & & & & & & & & \\
\hline Structure - West & 76680 & USACE & $\begin{array}{l}\text { \&fid =\&dt=S } \\
\text { http://rivergages.mvr.usac }\end{array}$ & 29.7578 & -92.2642 & 1/1/1961 & $1 / 27 / 2015$ & 54.07 & unknown & unknown & unknown & unknown & unknown & unknown \\
\hline $\begin{array}{l}\text { Schooner Bayou } \\
\text { Control } \\
\text { Structure - East }\end{array}$ & 76600 & USACE & $\begin{array}{l}\text { e.army.mil/WaterControl/s } \\
\text { tationinfo2.cfm?sid=76600 } \\
\text { \&fid=\&dt=S } \\
\text { http://rivergages.mvr.usac }\end{array}$ & 29.7578 & -92.2638 & $1 / 1 / 1955$ & $5 / 21 / 2014$ & 59.38 & unknown & unknown & unknown & unknown & unknown & unknown \\
\hline $\begin{array}{l}\text { IWW at Leland } \\
\text { Bowman Lock - } \\
\text { West }\end{array}$ & 76800 & USACE & $\begin{array}{l}\text { e.army.mil/WaterControl/s } \\
\text { tationinfo2.cfm?sid }=76800 \\
\text { \&fid }=\& d t=S\end{array}$ & 29.7870 & -92.2093 & $1 / 1 / 1951$ & 7/7/2013 & 62.51 & unknown & unknown & unknown & unknown & unknown & unknown \\
\hline $\begin{array}{l}\text { IWW at Leland } \\
\text { Bowman Lock - }\end{array}$ & & & $\begin{array}{l}\text { http:///rivergages.mvr.usac } \\
\text { e.army.mil/WaterControl/s } \\
\text { tationinfo2.cfm?sid=76720 }\end{array}$ & & & & & & & & & & & \\
\hline & 76720 & USACE & $\begin{array}{l}\text { \&fid=\&dt=S } \\
\text { http://rivergages.mvr.usac }\end{array}$ & 29.7852 & -92.2041 & 1/1/1959 & $11 / 18 / 2014$ & 55.88 & unknown & unknown & unknown & unknown & unknown & unknown \\
\hline $\begin{array}{l}\text { Atchatalaya Bay } \\
\text { at Eugene }\end{array}$ & & & $\begin{array}{l}\text { e.army.mili//Watercontrol/s } \\
\text { tationinfo2.cfm?sid=88600 }\end{array}$ & & & & & & & & & & & \\
\hline Mississippi River & 88600 & USACE & $\begin{array}{l}\text { \&fid=\&dt=S } \\
\text { http://rivergages.mvr.usac } \\
\text { e.army.mil/Watercontrol/s } \\
\text { tationinfo2.cfm?sid=01480 }\end{array}$ & 29.3791 & -91.3818 & 9/30/2008 & $10 / 22 / 2018$ & 10.06 & unknown & unknown & unknown & unknown & unknown & unknown \\
\hline at Venice & 01480 & USACE & $\begin{array}{l}\text { 8fid=\&dt=S } \\
\text { http://rivergages.mvr.usac } \\
\text { e.army.mil/WaterControl/s }\end{array}$ & 29.2758 & -89.3528 & 1/15/1953 & $12 / 31 / 2014$ & 61.96 & unknown & unknown & unknown & unknown & unknown & unknown \\
\hline $\begin{array}{l}\text { Mississippi River } \\
\text { at West Bay }\end{array}$ & 01515 & USACE & $\begin{array}{l}\text { tationinfo2.cfm?sid =01515 } \\
\text { \&fid =\&dt=s } \\
\text { http://rivergages.mvr.usac } \\
\text { e.army.mil/WaterControl/s }\end{array}$ & 29.2401 & -89.2984 & 2/20/2009 & $10 / 22 / 2018$ & 9.67 & unknown & unknown & unknown & unknown & unknown & unknown \\
\hline $\begin{array}{l}\text { Receiving Area - } \\
\text { Outflow }\end{array}$ & 01516 & USACE & $\begin{array}{l}\text { tationinfo2.cfm?sid }=01516 \\
\text { \&fid }=\& d t=S\end{array}$ & 29.2192 & -89.3151 & $8 / 14 / 2008$ & $10 / 22 / 2018$ & 10.19 & unknown & unknown & unknown & unknown & unknown & unknown \\
\hline
\end{tabular}




\begin{tabular}{|c|c|c|c|c|c|c|c|c|c|c|c|c|c|c|}
\hline $\begin{array}{l}\text { Mississippi River } \\
\text { at Head of } \\
\text { Passes }\end{array}$ & 01545 & USACE & $\begin{array}{l}\text { http:///rivergages.mvr.usac } \\
\text { e.army.mil/WaterControl/s } \\
\text { tationinfo2.cfm?sid=01545 } \\
\text { \&fid=\&dt=S }\end{array}$ & 29.1338 & -89.2465 & $4 / 11 / 2008$ & $10 / 22 / 2018$ & 10.53 & unknown & unknown & unknown & unknown & unknown & unknown \\
\hline $\begin{array}{l}\text { Mississippi River } \\
\text { Southwest Pass }\end{array}$ & & & $\begin{array}{l}\text { http:///rivergages.mvr.usac } \\
\text { e.army.mil/WaterControl/s } \\
\text { tationinfo2.cfm?sid }=01575\end{array}$ & & & & & & & & & & & \\
\hline Mile 7.5 BHP & 01575 & USACE & \& fid $=\& d t=S$ & 29.0564 & -89.3086 & $12 / 3 / 2010$ & $10 / 22 / 2018$ & 7.89 & unknown & unknown & unknown & unknown & unknown & unknown \\
\hline $\begin{array}{l}\text { Mississippi River } \\
\text { Southwest Pass }\end{array}$ & & & $\begin{array}{l}\text { http://rivergages.mvr.usac } \\
\text { e.army.mil/waterControl/s } \\
\text { tationinfo2.cfm?sid =01670 }\end{array}$ & & & & & & & & & & & \\
\hline $\begin{array}{l}\text { at East Jetty } \\
\text { Bayou Dupre } \\
\text { Sector Gate - } \\
\text { West/Protected }\end{array}$ & 01670 & USACE & 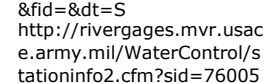 & 28.9323 & -89.4071 & 1/1/1953 & $7 / 7 / 2015$ & 62.51 & unknown & unknown & unknown & unknown & unknown & unknown \\
\hline Side & 76005 & USACE & 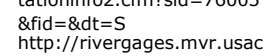 & 29.9346 & -89.8372 & $4 / 29 / 2008$ & $10 / 22 / 2018$ & 10.48 & unknown & unknown & unknown & unknown & unknown & unknown \\
\hline $\begin{array}{l}\text { Bayou Dupre } \\
\text { Sector Gate - }\end{array}$ & & & $\begin{array}{l}\text { e.army.mil/WaterControl/s } \\
\text { tationinfo2.cfm?sid }=76010\end{array}$ & & & & & & & & & & & \\
\hline $\begin{array}{l}\text { East/Flood Side } \\
\text { Bayou }\end{array}$ & 76010 & USACE & $\&$ fid $=\& d t=S$ & 29.9352 & -89.8366 & $4 / 29 / 2008$ & $10 / 22 / 2018$ & 10.48 & unknown & unknown & unknown & unknown & unknown & unknown \\
\hline $\begin{array}{l}\text { Bienvenue } \\
\text { Floodgate - } \\
\text { West/Protected }\end{array}$ & & & $\begin{array}{l}\text { http: ///rivergages.mvr.usac } \\
\text { e.army.mil/WaterControl/s } \\
\text { tationinfo2.cfm?sid= }=76024\end{array}$ & & & & & & & & & & & \\
\hline & 76024 & USACE & \&fid $=\& d t=S$ & 29.9978 & -89.9165 & $4 / 29 / 2008$ & $10 / 22 / 2018$ & 10.48 & unknown & unknown & unknown & unknown & unknown & unknown \\
\hline $\begin{array}{l}\text { Bayou } \\
\text { Bienvenue } \\
\text { Floodgate - }\end{array}$ & & & $\begin{array}{l}\text { http://rivergages.mvr.usac } \\
\text { e.army.mil/WaterControl/s } \\
\text { tationinfo2.cfm?sid=76025 }\end{array}$ & & & & & & & & & & & \\
\hline $\begin{array}{l}\text { East/Flood Side } \\
\text { IHNC Surge }\end{array}$ & 76025 & USACE & $\begin{array}{l}\text { \&fid=\&dt=S } \\
\text { http://rivergages.mvr.usac } \\
\text { e.army.mil/WaterControl/s }\end{array}$ & 29.9987 & -89.9153 & $1 / 1 / 2007$ & $10 / 22 / 2018$ & 11.81 & unknown & unknown & unknown & unknown & unknown & unknown \\
\hline $\begin{array}{l}\text { Barrier West / } \\
\text { Protected Side }\end{array}$ & 76032 & USACE & $\begin{array}{l}\text { tationinfo2.cfm?sid }=76032 \\
\text { \&fid =\&dt=S } \\
\text { http://rivergages.mvr.usac }\end{array}$ & 30.0124 & -89.9006 & $2 / 17 / 2010$ & $10 / 22 / 2018$ & 8.68 & unknown & unknown & unknown & unknown & unknown & unknown \\
\hline $\begin{array}{l}\text { IHNC Surge } \\
\text { Barrier East / } \\
\text { Flood Side }\end{array}$ & 76030 & USACE & $\begin{array}{l}\text { e.army.mil/WaterControl/s } \\
\text { tationinfo2.cfm?sid }=76030 \\
\& \text { fid }=\& d t=S\end{array}$ & 30.0124 & -89.9005 & $10 / 15 / 2010$ & $10 / 22 / 2018$ & 8.02 & unknown & unknown & unknown & unknown & unknown & \\
\hline $\begin{array}{l}\text { Chef Menteur } \\
\text { Pass nr Lake }\end{array}$ & 10030 & USACE & $\begin{array}{l}\text { http////ivergages.mvr.usac } \\
\text { e.army.mil/WaterControl/s } \\
\text { tationinfo2.cfm?sid }=85750\end{array}$ & 30.0124 & -09.9003 & $10 / 13 / 2010$ & $10 / 22 / 2010$ & 8.02 & . & . & . & . & . & Uniknown \\
\hline Borgne & 85750 & USACE & $\begin{array}{l}\text { \&fid =\&dt=S } \\
\text { http://rivergages.mvr.usac }\end{array}$ & 30.0667 & -89.8010 & $10 / 9 / 2007$ & $10 / 22 / 2018$ & 11.04 & unknown & unknown & unknown & unknown & unknown & unknown \\
\hline $\begin{array}{l}\text { Rigolets near } \\
\text { Lake }\end{array}$ & & & $\begin{array}{l}\text { e.army.mil/WaterControl/s } \\
\text { tationinfo2.cfm?sid }=85700\end{array}$ & & & & & & & & & & & \\
\hline $\begin{array}{l}\text { Pontchartrain } \\
\text { Lake }\end{array}$ & 85700 & USACE & $\begin{array}{l}\text { \&fid=\&dt=S } \\
\text { httpp////rivergages.mvr.usac } \\
\text { e.army.mil//WaterControl/s }\end{array}$ & 30.1634 & -89.7379 & $1 / 1 / 1950$ & $8 / 6 / 2001$ & 51.59 & unknown & unknown & unknown & unknown & unknown & unknown \\
\hline $\begin{array}{l}\text { Pontchartrain at } \\
\text { Mandeville } \\
\text { Lake }\end{array}$ & 85575 & USACE & $\begin{array}{l}\text { tationinfo2.cfm?sid }=85575 \\
\text { \&fid =\&dd=S } \\
\text { http://rivergages.mvr.usac }\end{array}$ & 30.3658 & -90.0923 & 8/2/1957 & $7 / 26 / 2002$ & 44.98 & unknown & unknown & unknown & unknown & unknown & unknown \\
\hline $\begin{array}{l}\text { Pontchartrain at } \\
\text { Lakefront } \\
\text { Airport }\end{array}$ & 85670 & USACE & $\begin{array}{l}\text { e.army.mil/WaterControl/s } \\
\text { tationinfo2.cfm?sid }=85670 \\
\text { \&fid }=\text { ddt }=\mathrm{S}\end{array}$ & & & & & & unknown & unknown & Junnown & unknown & unknown & unknown \\
\hline $\begin{array}{l}\text { Seabrook } \\
\text { Floodgate } \\
\text { Closure } \\
\text { Structure - Lake }\end{array}$ & $850 / 0$ & USACE & $\begin{array}{l}\text { \&fid=\&dt=S } \\
\text { http://rivergages.mvr.usac } \\
\text { e.army.mil/WaterControl/s } \\
\text { tationinfo2.cfm?sid }=76065\end{array}$ & 30.0399 & -90.0188 & $1 / 1 / 2007$ & $10 / 22 / 2018$ & 11.81 & unknown & unknown & unknown & . & . & Unknown \\
\hline $\begin{array}{l}\text { Side } \\
\text { Seabrook }\end{array}$ & 76065 & USACE & \&fid $=\& \mathrm{dt}=\mathrm{S}$ & 30.0304 & -90.0347 & $7 / 19 / 2012$ & $10 / 22 / 2018$ & 6.26 & unknown & unknown & unknown & unknown & unknown & unknown \\
\hline $\begin{array}{l}\text { Floodgate } \\
\text { Closure } \\
\text { Structure - }\end{array}$ & & & $\begin{array}{l}\text { http:///rivergages.mvr.usac } \\
\text { e.army.mil/WaterControl/s } \\
\text { tationinfo2.cfm?sid=76062 }\end{array}$ & & & & & & & & & & & \\
\hline $\begin{array}{l}\text { Seabrook Bridge } \\
\text { - Inner Harbor }\end{array}$ & 76062 & USACE & 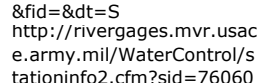 & 30.0302 & -90.0346 & $7 / 19 / 2012$ & $10 / 22 / 2018$ & 6.26 & unknown & unknown & unknown & unknown & unknown & unknown \\
\hline $\begin{array}{l}\text { Nav Canal } \\
\text { Bayou St John } \\
\text { at Lake }\end{array}$ & 76060 & USACE & $\begin{array}{l}\text { \&fid=\&dt=S } \\
\text { http:///rivergages.mvr.usac } \\
\text { e.army.mil/WaterControl/s } \\
\text { tationinfo2.cfm?sid=85634 }\end{array}$ & 30.0242 & -90.0313 & $5 / 21 / 2010$ & $10 / 22 / 2018$ & 8.42 & unknown & unknown & unknown & unknown & unknown & unknown \\
\hline $\begin{array}{l}\text { Pontchartrain } \\
\text { Lake } \\
\text { Pontchartrain at }\end{array}$ & 85634 & USACE & $\begin{array}{l}\text { \&fid=\&dt=S } \\
\text { http:///rivergages.mvr.usac } \\
\text { e.army.mil/WaterControl/s } \\
\text { tationinfo2.cfm?sid=85625 }\end{array}$ & 30.0243 & -90.0827 & $4 / 29 / 2011$ & $10 / 22 / 2018$ & 7.48 & unknown & unknown & unknown & unknown & unknown & unknown \\
\hline $\begin{array}{l}\text { West End } \\
\text { Whith de }\end{array}$ & 85625 & USACE & $\begin{array}{l}\text { \&fid }=\& d t=S \\
\text { s. }\end{array}$ & 30.0222 & -90.1156 & $3 / 10 / 1949$ & $12 / 31 / 2014$ & 65.81 & unknown & unknown & unknown & unknown & unknown & unknown \\
\hline
\end{tabular}




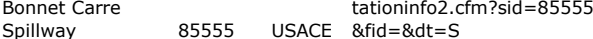
httpp ///rivergages.mvr.usac
e.army.mil/WaterControl/s

$\begin{array}{ll}\text { Pass Manchac } & \text { e.army.mil/WaterControl/s } \\ \text { near } & \text { tationinfo2.cfm?sid }=85420\end{array}$

tationinfo2.cfm?sid $=85420$
\&fid $=\& d t=S$

$\begin{array}{lcrl}\begin{array}{l}\text { Pontchatoula } \\ \text { Cattish Point }\end{array} & 85420 \quad \text { USACE } & \begin{array}{l}\text { \&fid=\&dt=S } \\ \text { nttp://rivergages.mur.usac } \\ \text { Control }\end{array} & \text { e.army.mil/WaterControl/s }\end{array}$

Structure -

North
Cattish Point

Control
Structure -

South
Inner Harbor

tationinfo2.cfm?sid =70675

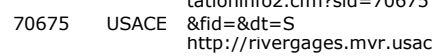

http://rivergages.mvr.us

70750 USACE $\begin{aligned} & \text { tationinfo2.cfm?sid } \& \text { sid }=80750 \\ & \text { add }=S\end{aligned}$

http://rivergages.mur.usac

Canal (IHNC

Lock) New tationinfo2. cfm?sid=76160

$\begin{aligned} & \\ & \text { http://rivergages.mvr.usac } \\ & \text { e.army.mil/WaterControl/s }\end{aligned}$

$\begin{array}{lll}\begin{array}{l}\text { Bayou Boeuf } \\ \text { Lock - East }\end{array} \quad 76360 \quad \text { USACE } & \begin{array}{l}\text { tationinfo2.cfm?sid } \\ \text { \&fid=\&dt=S }\end{array}\end{array}$

IWW at hetp://rivergages.mvr.usac

$\begin{array}{ll}\text { IWW at } & \text { e.army.mil/WaterControl/s } \\ \text { Calcasieu Lock - } & \text { tationinfo2.cfm?sid }=76960\end{array}$

$\begin{array}{lll}\text { Calcasieu Lock - } 76960 \quad \text { USACE } & \begin{array}{l}\text { tationinfo2.cfm?sid=76960 } \\ \text { \&fid=\&dt=S } \\ \text { hest }\end{array} \\ \text { http///rivergages.mvr.usac }\end{array}$

Mississippi River $\quad$ e.army.mil/WaterControl/s

$\begin{array}{lll}\begin{array}{l}\text { at New Orleans } \\ \text { (Carrollton) }\end{array} & 01300 \quad \text { USACE } & \begin{array}{l}\text { tationifolo. } 2 . c f m ? s i d=01300 \\ 8 \text { fid }=\& d t=S\end{array}\end{array}$

$\begin{aligned} 01300 \quad \text { USACE } & \begin{array}{l}\text { \&fid=\&dt=S } \\ \text { http://rivergages.mvr.usac } \\ \text { e.army.mil/WaterControl/s }\end{array} \\ & \text { (Carrolton) }\end{aligned}$

$\begin{array}{ll}\text { Mississippi River } & \text { e.army.mil/WaterControl/s } \\ \text { tationinfo2.cfm?sid }=01340\end{array}$

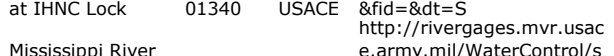

$\begin{array}{ll}\text { Mississippi River } & \text { e.army.mil/WaterControl/s } \\ \text { at West Pointe a } & \text { tationinfo2.cfm?sid }=01400\end{array}$

$\begin{array}{llll}0 & \text { la Hache } 01400 \quad \text { USACE } & \begin{array}{l}\text { \&fid=\&dt=S } \\ \text { http://rivergages.mvr.usac }\end{array}\end{array}$

$\begin{array}{ll}\text { Calcasieu River } & \text { e.army.mil/WaterControl/s } \\ \text { \& Pass at Lake } & \text { tationinfo2 }\end{array}$

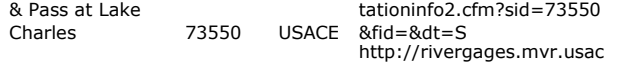

$\begin{array}{ll}\text { GIWW at } & \text { e.army.mil/WaterControl/s } \\ \text { tationinfo2.cfm?sid }=76320\end{array}$

Houma
Houma

$\begin{aligned} & \text { Bayou Lafourche } \\ & \text { at Leeville }\end{aligned}$
$82350 \quad$ USACE

Bayou Barataria
at Barataria

$\begin{array}{llll}\text { at Barataria } & 82750 & \text { USACE } & \\ \text { nttp:///rivergages.mur.usac } & \\ \text { e.army.mil/WaterControl/s }\end{array}$

$\begin{array}{ll}\text { Bayou Terre Aux } & \text { tationinfo2.cfm?sid }=85780\end{array}$

$\begin{array}{llll}\begin{array}{l}\text { Delacroix, La. } \\ \text { MRGO at Shell }\end{array} & 85780 & \text { USACE } & \text { \& fatid }=\& d \mathrm{dt}=S \\ \text { Beach } & 85800 & \text { USACE }\end{array}$

Beach $85800 \quad$ USACE ${ }_{\text {http://nwis.waterdata.usgs }}$ gov/nwis/inventory/?site

$\begin{array}{lll}\text { Calcasieu River } 08017118 \text { USGS } & \begin{array}{l}\text { no }=08017118 \& \text { agency_cd } \\ =\end{array} \text { USGS }\end{array}$

$07381349 \quad$ http://waterdata.usgs.gov/

$\begin{array}{llll}\text { Caillou Bay } & \begin{array}{l}07381349 \\ 8\end{array} & \text { USGS } & \begin{array}{l}\text { la/nwis/uv/?site_no=0738 } \\ 134988 \text { \&agency_cd=USGS }\end{array}\end{array}$

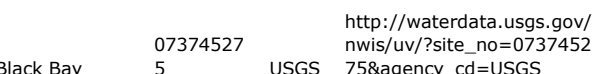

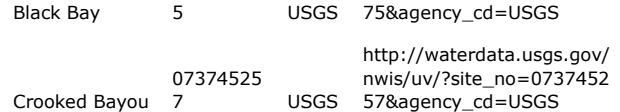

$\begin{array}{llllll}30.0559 & -90.3722 & 1 / 1 / 2007 & 5 / 20 / 2011 & 4.38\end{array}$

$\begin{array}{llllll}30.2814 & -90.4003 & 4 / 17 / 2009 & 10 / 22 / 2018 & 9.51 & \text { unknown }\end{array}$

$29.8636 \quad-92.8484 \quad 1 / 1 / 1955 \quad 3 / 12 / 2015 \quad 60.19 \quad$ unknown

$29.8629 \quad-92.8492 \quad 1 / 1 / 1955 \quad 3 / 12 / 2015 \quad 60.19 \quad$ unknown

$29.9665 \quad-90.0268 \quad 1 / 1 / 1945 \quad 3 / 12 / 2015 \quad 70.19 \quad$ unknown

$\begin{array}{llllll}29.6830 & -91.1711 & 9 / 8 / 1954 & 3 / 12 / 2015 & 60.51 & \text { unknown }\end{array}$

$30.0887 \quad-93.2949 \quad 1 / 1 / 1951 \quad 8 / 27 / 2014 \quad 63.65 \quad$ unknown

$29.9347 \quad-90.1361 \quad 1 / 1 / 1950 \quad 1 / 1 / 2015 \quad 65.00 \quad$ unknown

$29.9644 \quad-90.0274 \quad 1 / 1 / 1945 \quad 1 / 27 / 2015 \quad 70.07 \quad$ unknown

$29.5711 \quad-89.7969 \quad 1 / 1 / 1950 \quad 12 / 31 / 2014 \quad 65.00 \quad$ unknown

$\begin{array}{llllll}30.2131 & -93.2569 & 1 / 1 / 1950 & 7 / 7 / 2003 & 53.51 & \text { unknown }\end{array}$

$\begin{array}{llllll}29.5981 & -90.7100 & 1 / 1 / 1959 & 11 / 17 / 2008 & 49.88 & \text { unknown }\end{array}$

$29.2478 \quad-90.2089 \quad 11 / 4 / 1955 \quad 4 / 8 / 2000 \quad 44.43 \quad$ unknown

$9.7414-90.1322 \quad 1 / 6 / 1950 \quad 11 / 12 / 1992 \quad 42.85 \quad$ unknown

$\begin{array}{llllll}29.7639 & -89.7922 & 5 / 15 / 1975 & 8 / 28 / 2005 & 30.29 & \text { unknown }\end{array}$

$29.8500 \quad-89.6833 \quad 6 / 30 / 1961 \quad 12 / 16 / 2002 \quad 41.46 \quad$ unknown

$29.8156 \quad-93.3489 \quad 10 / 1 / 2007 \quad 10 / 22 / 2018 \quad 11.06 \quad$ unknown

$29.0781 \quad-90.8714 \quad 10 / 1 / 2007 \quad 10 / 22 / 2018 \quad 11.06 \quad$ unknown

$\begin{array}{llllll}29.5693 & -89.5122 & 5 / 21 / 2014 & 10 / 22 / 2018 & 4.42 & \text { unknown }\end{array}$

unknown

unknown

unknown

unknown 


\begin{tabular}{|c|c|c|c|c|c|c|c|c|c|c|c|c|c|c|}
\hline Cow Bavou at & 07374525 & & $\begin{array}{l}\text { http://waterdata.usgs.gov/ } \\
\text { nwistipytor/?site }\end{array}$ & & & & & & & & & & & \\
\hline $\begin{array}{l}\text { Barataria Pass } \\
\text { at Grand Isle }\end{array}$ & $\begin{array}{l}07380251 \\
6\end{array}$ & USGS & $\begin{array}{l}73745258 \\
\text { http://waterdata.usgs.gov/ } \\
\text { nwis/uv/?site_no=0 } 0738025 \\
\text { 16\&agency_cd=USGS }\end{array}$ & 29.5706 & -89.7039 & $1 / 28 / 1999$ & $10 / 22 / 2018$ & 19.73 & unknown & $\begin{array}{l}\text { concrete retaining } \\
\text { wall }\end{array}$ & unknown & unknown & unknown & $\begin{array}{l}\text { unknown } \\
\text { unknown } \\
\text { (primary } \\
\text { benchmark } \\
\text { not } \\
\text { specified) }\end{array}$ \\
\hline $\begin{array}{l}\text { Mouth of } \\
\text { Atchafalaya } \\
\text { River } \\
\text { Crewboat }\end{array}$ & $\begin{array}{l}07381652 \\
5\end{array}$ & USGS & $\begin{array}{l}\text { http://waterdata.usgs.gov/ } \\
\text { nwis/uv/?site_no }=0738165 \\
\text { 25\&agency_dd=USGS } \\
\text { http://waterdata.usgs.gov/ } \\
\text { nwis/inventory/??ite no }=0\end{array}$ & 29.4303 & -91.3339 & $5 / 21 / 2015$ & $10 / 22 / 2018$ & 3.42 & unknown & unknown & unknown & unknown & unknown & unknown \\
\hline $\begin{array}{l}\text { Channel at Wax } \\
\text { Lake Outlet }\end{array}$ & $\begin{array}{l}07381592 \\
5\end{array}$ & USGS & $\begin{array}{l}\text { 73815925\&agency_cd =US } \\
\text { GS }\end{array}$ & 29.5400 & -91.4356 & $10 / 1 / 2002$ & $10 / 22 / 2018$ & 16.06 & unknown & unknown & unknown & unknown & unknown & unknown \\
\hline Vermilion Bay & 07387040 & USGS & $\begin{array}{l}\text { http:///waterdata.usgs.gov/ } \\
\text { nwis/uv/?site_no }=0738704 \\
\text { O\&agency_cd =USGS }\end{array}$ & 29.7131 & -91.8803 & 10/23/1997 & $10 / 22 / 2018$ & 21.00 & unknown & unknown & unknown & unknown & unknown & unknown \\
\hline $\begin{array}{l}\text { Vermilion Bay } \\
\text { Bayou Fearman }\end{array}$ & 07387050 & USGS & $\begin{array}{l}\text { http: ///waterdata.usgs.gov/ } \\
\text { nwis/dv/?site_no=0738705 } \\
\text { 08agenc_cd=USGS\&amp; } \\
\text { referred_module=sw }\end{array}$ & 29.6744 & -92.1356 & $10 / 1 / 1999$ & $10 / 22 / 2018$ & 19.06 & unknown & unknown & unknown & unknown & unknown & unknown \\
\hline $\begin{array}{l}\text { Lower } \\
\text { Atchafalaya } \\
\text { River at Morgan }\end{array}$ & & & $\begin{array}{l}\text { http://waterdata.usgs.gov/ } \\
\text { nwis/uv/?site_no }=0738160\end{array}$ & & & & & & & & & & & \\
\hline City & 07381600 & USGS & $\begin{array}{l}\text { O\&agency_cd=USGS } \\
\text { http://waterdata.usgs.gov/ }\end{array}$ & 29.6926 & -91.2118 & 10/1/1992 & 9/30/2015 & 23.00 & unknown & unknown & unknown & unknown & unknown & unknown \\
\hline $\begin{array}{l}\text { Lake Outlet) } \\
\text { Lake }\end{array}$ & 07381590 & USGS & $\begin{array}{l}\text { nwis/uv/?site_no=0738159 } \\
0\end{array}$ & 29.6978 & -91.3728 & 10/1/1995 & 9/30/2015 & 20.00 & unknown & unknown & unknown & unknown & unknown & unknown \\
\hline $\begin{array}{l}\text { North Calcasieu } \\
\text { Lake }\end{array}$ & & & $\begin{array}{l}\text { http://waterdata.usgs.gov/ } \\
\text { la/nwis/uv/?site_no }=0801 \\
\text { 7095\&PARAmeter_cd }=000\end{array}$ & & & & & & & & & & & \\
\hline (Hackberry) & 08017095 & USGS & $65,72020,63160,00060$ & 30.0317 & -93.2994 & $7 / 26 / 1997$ & $10 / 22 / 2018$ & 21.24 & unknown & unknown & unknown & unknown & unknown & unknown \\
\hline
\end{tabular}


Table S2. Information on 17 GNSS stations in coastal Louisiana.

\begin{tabular}{|c|c|c|c|c|c|}
\hline $\begin{array}{l}\text { GNSS } \\
\text { station code }\end{array}$ & Latitude & Longitude & $\begin{array}{l}\text { Foundation } \\
\text { depth (m) }\end{array}$ & $\begin{array}{l}\text { Depth to } \\
\text { Pleistocene } \\
\text { surface }(\mathrm{m})\end{array}$ & $\begin{array}{l}\text { Benchmark } \\
\text { foundation height } \\
\text { above Pleistocene } \\
\text { surface }(\mathrm{m})\end{array}$ \\
\hline AWES & 30.10 & -90.98 & 1 & 29 & 28 \\
\hline BVHS & 29.34 & -89.41 & $>20$ & 62 & $<42$ \\
\hline CAMR & 29.80 & -93.33 & unknown & & \\
\hline DSTR & 29.96 & -90.38 & unknown & & \\
\hline ENG1 & 29.88 & -89.94 & $\sim 3$ & 27 & $\sim 24$ \\
\hline ENG2 & 29.88 & -89.94 & $\sim 3$ & 27 & $\sim 24$ \\
\hline FRAN & 29.80 & -91.53 & 14.7 & 10 & Set in Pleistocene \\
\hline FSHS & 29.81 & -91.50 & 1 & 15 & 14 \\
\hline GRIS & 29.27 & -89.96 & unknown & & \\
\hline HOMA & 29.57 & -90.76 & 18.3 & 40 & 21.7 \\
\hline HOUM & 29.59 & -90.72 & $>15$ & 40 & $<25$ \\
\hline LAFR & 29.79 & -91.50 & unknown & & \\
\hline LMCN & 29.25 & -90.66 & 36.5 & 57 & 20.5 \\
\hline LWES & 29.90 & -90.35 & unknown & & \\
\hline MSSC & 30.38 & -89.61 & unknown & & \\
\hline NDBC & 30.36 & -89.61 & unknown & & \\
\hline VENI & 29.28 & -89.36 & 30.5 & 78 & 47.5 \\
\hline
\end{tabular}

